\title{
Effects of Input Data Uncertainties on an Air Traffic Control Difficulty Index
}

\author{
Sakae NAGAOKA ${ }^{\dagger}$, Fellow, Mark BROWN ${ }^{\dagger}$, Nonmember and Daniel DELAHAYE ${ }^{\dagger \dagger}$, Nonmember
}

SUMMARY Air traffic management (ATM) systems around the world are being modernized to accommodate shifts towards performance- and trajectory-based operations. These shifts will require new indices for safety, efficiency and complexity. The authors have been developing an index for evaluating air traffic control (ATC) difficulty that utilizes the relative positions and velocity vectors of aircraft pairs as input data. Prior to practical application of the index, it is necessary to understand the effects of input data error, i.e. errors in the positions and velocities of a pair of aircraft, on the estimated difficulty value. Two sensitivity analyses were therefore performed for a pair of aircraft cruising at constant speeds on intersecting linear tracks at the same altitude. Sensitivity analysis examines how uncertainty in inputs relates to uncertainty in outputs. Firstly, an analysis of propagation error was carried out. The formula of the propagation error at a certain point was derived based on the assumed input error, and the distribution of propagation error was investigated for all possible situations and compared with the distribution of difficulty values to clarify its characteristics. Secondly, a sensitivity analysis based on variance was carried out that evaluated the effect of each input parameter using a conditional variance value called the Sobol indices. Using a Monte Carlo method, we investigated the effect of each input parameter on the calculated difficulty value for all possible situations of aircraft pairs on intersecting trajectories. As a result, it was found that the parameter that most affects the difficulty value is the intersection angle of the trajectories.

key words: Air traffic control, Complexity, ATC difficulty, Uncertainty, Sensitivity analysis

\section{Introduction}

Air traffic management (ATM) systems around the world are being modernized to increase ATM capacity and efficiency through the adoption of performance- and trajectory-based aircraft operations [1]. In a performance-based environment, operational decisionmaking requires metrics of performance such as safety and efficiency appropriate to their application. Air traffic controller (ATCo) workload has a direct effect on ATM capacity and safety, but is hard to define and difficult to measure directly in operational environments. Various air traffic complexity and air traffic control (ATC) difficulty indices have therefore been proposed [2-11] that can be used as surrogates for workload. The estimation

$\dagger$ The author is with Electronic Navigation Research Institute(ENRI), Chofu-shi, 182-0012 Tokyo, Japan.

$\dagger+$ The Author is with Ecole National de l'Aviation Civile, 7 Ave. Edouard Belin, Toulouse, 34100 France methodologies of these metrics range from using only traffic data to those requiring subjective judgements of air traffic controllers. Most of these methods are not easily available for evaluation because the calculations or data collection required for estimation are not easy.

Because ATCo subjective judgement can only be applied a posteriori, it cannot be used by metrics intending to evaluate current or future system performance for tactical decision making. Many traffic complexity and ATC difficulty indices are therefore based on analysis of real-time trajectory sources data such as radar data. However, most existing approaches evaluate only the current traffic situation, so there is no index that evaluates the evolution of the spatial situation with time. The authors have therefore proposed as a new simple metric a difficulty index of air traffic control [1015] that takes into account future proximity situations based on current and planned trajectory information. The metric was expanded from a pairwise index to airspacewise index and calculation methods were developed for predicting future trajectory using flight plan information $[12,14]$.

We have hitherto focused on developing and improving the proposed methodology with a view to application in tactical ATM decision making [15], but the effects of uncertainty in the input data used by the metric had not been analyzed. To grasp these effects, we studied the metric's sensitivity to input parameters and the effects of measurement errors in the input data (i.e. position and velocity measurement errors of a surveillance system). For this purpose, sensitivity analyses [17-19] on the effects were carried out using two different approaches. The first approach was to analyze how input errors propagate to the calculated difficulty index in an encounter situation between an aircraft pair. The second was to analyze the sensitivity of the difficulty index to each input parameter for all possible encounter situations. These analyses are based on cruise flight phase model with the encounter situation of aircraft pairs in level flight at a constant speed and heading. Numerical results for a typical case are presented.

The remainder of this paper is organized as follows: Section 2 provides details about the difficulty index and its calculation; Sections 3 and 4 respectively describe the 
formula for calculating propagation errors and present examples of calculation; Section 5 deals with global sensitivity analysis for parameters relating to encounter situations; Section 6 summarizes the paper.

\section{Difficulty Index and Its Calculation}

\subsection{Air Traffic Control and Proximity}

The air traffic control service is provided to prevent collisions and to ensure a safe, orderly and expeditious flow of air traffic [20]. Separation minima are designated for safe air traffic operations in various types of airspace. Air traffic controllers intervene to prevent proximity situations called conflicts. A conflict is an event in which two or more aircraft experience, or are predicted to experience, a "loss of separation" that is, proximity less than the prescribed minima.

When dealing with conflicts from an ATCo workload perspective, we focused on the proximity situations of pairs of aircraft. To construct a difficulty index, we tried to use the distance and the remaining time to the closest point of approach (CPA) between the aircraft pair as essential parameters of the index [10-11].

ATCo decision-making is based on the present and predicted air traffic situations. We hypothesize that perceived need, and hence a predicted proximity event, induces a workload that depends on the severity of the proximity event (that is, minimum distance at CPA) and the time to remaining to resolve it by intervention. Therefore, we adopt such proximity situations as a measure of air traffic control difficulty. We proposed a difficulty index based on the proximity situations of air traffic flying in en-route airspace where linear extrapolation of trajectory was largely applicable. The proposed difficulty value presented in the following section can be estimated using trajectory information extracted from surveillance radar data.

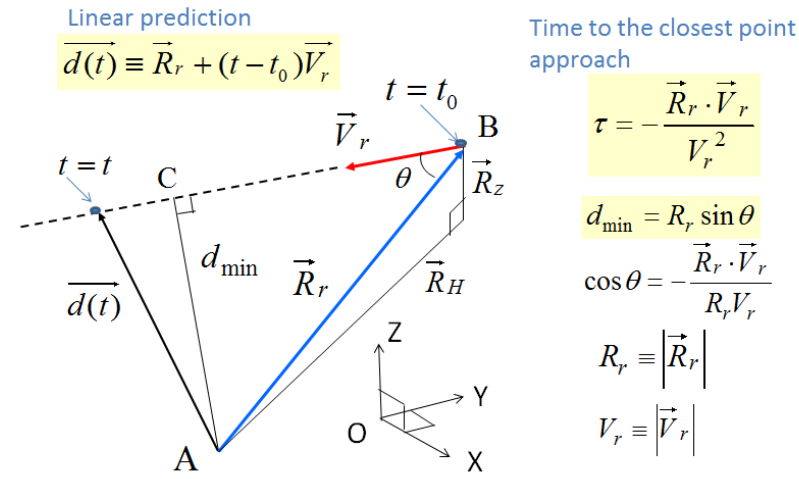

Fig.1 Geometric configuration of relative motion of aircraft

\subsection{Difficulty Index and Its Calculation}

We define an ATC difficulty value for a pair of aircraft at a given time $t_{0}$ (Fig.1) by the following equation:

$$
G\left(t_{0}\right)=\max _{t \geq t_{0}} \exp [-C(t)]=\exp \left[-\min _{t \geq t_{0}} C(t)\right]
$$

where $C(t)$ is an objective function. The difficulty value at time $t=t_{0}, G\left(t_{0}\right)$ is the value $\exp \left[-C\left(t_{\min }\right)\right]$ at the projected time $t_{\min }$ which minimizes the following objective function:

$$
C(t) \equiv \frac{\mathrm{R}_{H}(t)^{2}}{\lambda_{H}{ }^{2}}+\frac{\mathrm{R}_{z}(t)^{2}}{\lambda_{z}{ }^{2}}+\frac{\left(t-t_{0}\right)^{2}}{\lambda_{t}{ }^{2}}
$$

where $R_{H}(t)$ is the relative horizontal distance between the aircraft pair at time $t$ and $R_{z}(t)$ is the relative vertical distance. The third term on the right hand side (RHS) of Eq. (2) is the temporal distance from the observation time to the projected time. $\lambda_{H}, \lambda_{z}, \lambda_{t}$ are scale parameters of the horizontal, vertical and temporal dimensions, respectively.

Eq. (2) can be rewritten as the following four dimensional (4D) vector representation:

$$
C(t)=\left|\overrightarrow{R_{r}^{*}}(t)\right|^{2} / \lambda_{H}{ }^{2} \quad \text { for } \quad t \geq t_{0}
$$

and $\overrightarrow{R_{r}^{*}}(t)$ can be estimated using the following 4D vectors and scalers [14]:

$$
\begin{aligned}
& \overrightarrow{R_{r}^{*}}\left(t_{0}\right)=\left[x_{r}, y_{r}, k_{z} z_{r}, 0\right]^{T} \equiv \overrightarrow{R_{r}^{*}} \\
& \overrightarrow{V_{r}^{*}}\left(t_{0}\right)=\left[v_{r_{x}}, v_{r_{y}}, k_{z} v_{r_{z}}, k_{t}\right]^{T} \equiv \overrightarrow{V_{r}^{*}} \\
& k_{z}=\lambda_{H} / \lambda_{z} \\
& k_{t}=\lambda_{H} / \lambda_{t}
\end{aligned}
$$

where $x_{r}, y_{r}, z_{r}$ are the coordinate axis components of the relative position vector of the pair at observation time $t_{0}$, and $v_{r_{x}}, v_{r_{y}}$ and $v_{r_{z}}$ are respectively the coordinate components of the relative velocity vector at the time $t_{0}$. By linear extrapolation of these values, the projected 4D vector at time $t$ is assumed to be given by

$$
\overrightarrow{R_{r}^{*}}(t)=\overrightarrow{R_{r}^{*}}\left(t_{0}\right)+\overrightarrow{V_{r}^{*}}\left(t_{0}\right) \cdot\left(t-t_{0}\right)
$$

Then , Eq. (3) becomes

$$
C(t)=\frac{\left[\overrightarrow{R_{r}^{*}}+\overrightarrow{V_{r}^{*}} \cdot\left(t-t_{0}\right)\right]^{2}}{\lambda_{H}{ }^{2}}
$$

Differentiating Eq. (9), the time which minimizes the objective function is given by

$$
t_{\text {min }}=t_{0}-\frac{\left(\overrightarrow{\left.R_{r}^{*} \cdot \overrightarrow{V_{r}^{*}}\right)}\right.}{\left|\overrightarrow{V_{r}^{*}}\right|^{2}}
$$

Defining the elapsed time from $t_{0}$ to $t_{\min }$ as

$$
\tau \equiv t_{\min }-t_{0}
$$

the minimum value of the objective function is given by 


$$
\min _{t \geq t_{0}} C(t)=\left\{\begin{array}{ccc}
C\left(t_{0}\right) & \text { for } & \tau<0 \\
C\left(t_{\text {min }}\right) & \text { for } & \tau \geq 0
\end{array}\right.
$$

This yields the difficulty value $G\left(t_{0}\right)$ as

$$
G\left(t_{0}\right)=\left\{\begin{array}{cc}
\exp \left(-\left|\overrightarrow{R_{r}^{*}}\right|^{2} / \lambda_{H}{ }^{2}\right) & \text { for } \quad \tau \leq 0 \\
\exp \left\{-\frac{1}{\lambda_{H}{ }^{2}}\left[\left|\overrightarrow{R_{r}^{*}}\right|^{2}-\frac{\left(\overrightarrow{\left.R_{r}^{*} \cdot \overrightarrow{V_{r}^{*}}\right)^{2}}\right.}{\left|\overrightarrow{V_{r}^{*}}\right|^{2}}\right]\right\} \quad \text { for } \tau>0
\end{array}\right.
$$

where

$$
\begin{aligned}
& \left|\overrightarrow{R_{r}^{*}}\right|^{2}=x_{r}^{2}+y_{r}^{2}+k_{z}^{2} z_{r}^{2} \\
& \left|\overrightarrow{V_{r}^{*}}\right|^{2}=v_{r_{x}}^{2}+v_{r_{y}}{ }^{2}+k_{z}^{2} v_{r_{z}}^{2}+k_{t}^{2} \\
& \left(\overrightarrow{R_{r}^{*}} \cdot \overrightarrow{V_{r}^{*}}\right)=x_{r} v_{r_{x}}+y_{r} v_{r_{y}}+k_{z}^{2} z_{r} v_{r_{z}}
\end{aligned}
$$

\section{Error Propagation to Difficulty Value}

\subsection{Error Propagation Equation}

This section derives equations which relate errors in the input data to the error in the difficulty value $G\left(t_{0}\right)$ calculated using the input data; that is, how errors in the observed relative position and velocity vectors at time $t_{0}$ propagate to $G\left(t_{0}\right)$.

The $G\left(t_{0}\right)$ of Eq.(13) is a function of the components of two vectors (Eqs. (4) and (5)), i.e.,

$$
G\left(x_{r}, y_{r}, z_{r}, v_{r_{x}}, v_{r_{y}}, v_{r_{z}}\right) \equiv G\left(t_{0}\right)
$$

Let us denote

$$
F\left(x_{r}, y_{r}, z_{r}, v_{r_{x}}, v_{r_{y}}, v_{r_{z}}\right) \equiv-\lambda_{H}^{2} \ln G\left(t_{0}\right)
$$

Also denote the observed variables $\left[x_{r}, y_{r}, z_{r}, v_{r_{x}}, v_{r_{v}}, v_{r_{z}}\right]$ by $\underline{u}=\left[u_{1}, u_{2}, . ., u_{6}\right]$ and their errors by $\underline{\varepsilon}=\left[\varepsilon_{u_{1}}, \varepsilon_{u_{2}}, . ., \varepsilon_{u_{6}}\right]$. These values are for a pair of aircraft and consist of the difference between their position and velocity observations. The following approximation for the bias $\varepsilon_{G}$ and variance $\sigma_{G}{ }^{2}$ of the propagation errors can be obtained. (This approximation can be derived using the Taylor expansion of an observed value around the true value, and taking only the $1^{\text {st }}$-order terms assuming that errors of each component are independently distributed.)

$$
\begin{aligned}
& \mathcal{E}_{G} \approx \sum_{i=1}^{6}\left(\frac{\partial G}{\partial u_{i}}\right)_{t=t_{0}} \varepsilon_{u_{i}} \\
& \sigma_{G}^{2}=V\left(\varepsilon_{G}\right) \approx \sum_{i=1}^{6}\left(\frac{\partial G}{\partial u_{i}}\right)_{t=t_{0}}^{2} V\left(\varepsilon_{u_{i}}\right)
\end{aligned}
$$

where $V\left(\varepsilon_{u_{i}}\right)$ is the variance of $u_{i} \cdot(\mathrm{i}=1, . ., 6)$.
Table 1. Partial derivatives of $F \quad\left(\tau \equiv-\left(\overrightarrow{R_{r}^{*}} \cdot \overrightarrow{V_{r}^{*}}\right) /\left|\overrightarrow{V_{r}^{*}}\right|^{2}\right)$

\begin{tabular}{|c|c|c|}
\hline $\begin{array}{c}\text { Variable } \\
u_{i}\end{array}$ & $\begin{array}{c}\partial F / \partial u_{i} \\
\text { for } \tau<0\end{array}$ & $\begin{array}{c}\partial F / \partial u_{i} \\
\text { for } \tau \geq 0\end{array}$ \\
\hline$u_{1}=x_{r}$ & $2 x_{r}$ & $2\left(x_{r}+v_{r_{x}} \tau\right)$ \\
\hline$u_{2}=y_{r}$ & $2 y_{r}$ & $2\left(y_{r}+v_{r_{y}} \tau\right)$ \\
\hline$u_{3}=z_{r}$ & $2 z_{r} k_{z}^{2}$ & $2\left(z_{r}+v_{r_{z}} \tau\right) k_{z}^{2}$ \\
\hline$u_{4}=v_{r x}$ & 0 & $2\left(x_{r}+v_{r_{x}} \tau\right) \tau$ \\
\hline$u_{5}=v_{r y}$ & 0 & $2\left(y_{r}+v_{r_{y}} \tau\right) \tau$ \\
\hline$u_{6}=v_{r z}$ & 0 & $2\left(z_{r}+v_{r_{z}} \tau\right) \tau k_{z}^{2}$ \\
\hline
\end{tabular}

\subsection{Derivation of Formula for Propagation Error}

From Eq. (20), the partial derivative of $G$ by a variable $u$ is given by

$$
\frac{\partial G}{\partial u}=-\frac{e^{-F / \lambda_{H}^{2}}}{\lambda_{H}^{2}} \frac{\partial F}{\partial u}
$$

Since the exponential part is equal to $G$, this equation consists of the product of the partial derivatives of $F$ and $G$.

Table 1 shows derived partial derivatives of the function $F$. Herein, $\tau$ is the elapsed time from $t_{0}$ to $t_{\min }$.

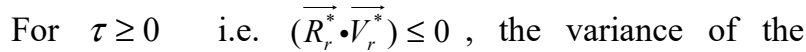
propagation errors of Eq. (20) is given by

$$
\begin{aligned}
\sigma_{G}^{2} & \simeq 4\left(\frac{e^{-F / \lambda_{H}{ }^{2}}}{\lambda_{H}{ }^{2}}\right)^{2} \times \\
& \left\{\left(x_{r}+v_{r x} \tau\right)^{2} V\left(\varepsilon_{u_{1}}\right)+\left(y_{r}+v_{r y} \tau\right)^{2} V\left(\varepsilon_{u_{2}}\right)\right. \\
& +k_{z}{ }^{4}\left(z_{r}+v_{r z} \tau\right)^{2} V\left(\varepsilon_{u_{3}}\right)+\tau^{2}\left(x_{r}+v_{r x} \tau\right)^{2} V\left(\varepsilon_{u_{4}}\right) \\
& \left.+\tau^{2}\left(y_{r}+v_{r y} \tau\right)^{2} V\left(\varepsilon_{u_{5}}\right)+k_{z}{ }^{4} \tau^{2}\left(z_{r}+v_{r z} \tau\right)^{2} V\left(\varepsilon_{u_{6}}\right)\right\}
\end{aligned}
$$

where $V\left(\varepsilon_{u_{i}}\right)$ is the variance of the error $\varepsilon_{u_{i}}$. This equation can be calculated from the relative position and velocity observed at time $t_{0}$, and the estimated values of their measurement errors. For $\tau<0$, Eq. (20) is identical to Eq. (22) into which $\tau=0$ is substituted.

\subsection{Estimating Uncertainty of Input Data}

\subsubsection{Errors of Input Data}

The input data for calculating the difficulty index is usually derived by estimating the relative position and relative velocity of a pair of aircraft from observations of each aircraft obtained by a surveillance radar system. In typical air traffic control air route surveillance, position measurements from several radars are combined to give a single observation for each aircraft [16] and for air traffic control purposes, it is typically assumed that measurement 
error is uniform throughout the surveillance coverage volume but in practice this is not the case. The measurement (estimation) error of single aircraft by a single radar varies depending on the geometrical relationship between the aircraft and the sensor (i.e. the radar antenna). When considering the relative positions of a pair of aircraft, the bias components may be cancelled out if measurement errors are highly correlated. Measurement errors may be close to uncorrelated if the pair of aircraft pairs are far enough apart. Herein, as a preliminary analysis, we assume that the error in each direction in the plane is independently distributed with zero mean and constant variance (e.g. the maximum value for the observed airspace) so that the error can be easily handled and the estimation is not underestimated.

\subsubsection{Estimation of velocities}

We assume position data extracted from a radar surveillance system as inputs $\left[\hat{x}_{i}, \hat{y}_{i}, \hat{z}_{i}, \hat{t}_{i}\right](i=1,2, \ldots, n)$. The errors of these inputs $\left[e_{x, i}, e_{y, i}, e_{z, i}, e_{t, i}\right]$ depend on data processing methods. Aircraft velocities can be estimated by smoothing consecutive position samples. Here, we deal with the simplest case, assuming that consecutive position samples are acquired with a constant sampling period of $T=10 \mathrm{~s}$. For instance, using position data of $i$-th and $(i+1)$-th periods, we estimate the $X$ component of aircraft velocity in the $(\mathrm{i}+1)$-th period, $v_{x}$ as

$$
\hat{v}_{x}=\frac{\hat{x}_{i+1}-\hat{x}_{i}}{\hat{t}_{i+1}-\hat{t}_{i}}
$$

The estimation error of the velocity component $\varepsilon_{v_{x}}$ can be approximated by

$$
\varepsilon_{v_{x}} \equiv \hat{v}_{x}-v_{x} \approx \frac{\left(e_{x, i+1}-e_{x, i}\right)}{\left(t_{i+1}-t_{i}\right)}-\frac{\left(e_{t, i+1}-e_{t, i}\right) v_{x}}{\left(t_{i+1}-t_{i}\right)}
$$

We assume that the errors in the $X, Y, Z$ and $t$ dimensions are mutually independent, have zero means, and have variances $\sigma_{x}^{2}, \sigma_{y}^{2}, \sigma_{z}^{2}$ and $\sigma_{t}^{2}$ respectively.

Since the observation intervals are almost constant, that is $t_{i+1}-t_{i} \approx T$, the variance $\sigma_{v x}{ }^{2} \equiv V\left(\varepsilon_{v x}\right)$ of velocity error in the $X$ direction $\varepsilon_{v x}$ can be approximated by

$$
{\sigma_{v_{x}}}^{2}=\frac{2}{T^{2}}\left(\sigma_{x}^{2}+\hat{v}_{x}^{2} \sigma_{t}^{2}\right)
$$

and similar approximations are obtained for the $Y$ and $Z$ directions

$$
\begin{aligned}
& {\sigma_{v_{y}}}^{2}=\frac{2}{T^{2}}\left({\sigma_{y}}^{2}+\hat{v}_{y}^{2} \sigma_{t}^{2}\right) \\
& \sigma_{v_{z}}{ }^{2}=\frac{2}{T^{2}}\left(\sigma_{z}^{2}+\hat{v}_{z}^{2} \sigma_{t}^{2}\right)
\end{aligned}
$$

3.3.3 Errors in Relative Position and Velocity

Assuming that data of aircraft A and aircraft B are obtained by the same measurement system and that error components in each spatial dimension $X, Y, Z$ are independent and have the same variances, the variance of each component of relative position error can be approximated by

$$
\begin{aligned}
& {\sigma_{r x}}^{2}=\sigma_{x, A}^{2}+\sigma_{x, B}^{2} \approx 2 \sigma_{x}{ }^{2} \\
& \sigma_{r y}{ }^{2}=2 \sigma_{y}{ }^{2} \\
& \sigma_{r z}^{2}=2 \sigma_{z}^{2}
\end{aligned}
$$

The subscripts $A$ and $B$ correspond to aircraft A and aircraft $\mathrm{B}$, respectively.

Variances of relative velocity error vary according to the geometric relationships between the pair of aircraft since these depend on the velocity components of the relative positions, as shown in from Eqs. (25)-(27).

Herein, for simplicity, we replace the speed $\left|\hat{v}_{u}\right|(u$ means $x, y$ or $z$ ) by an upper bound speed $v_{\max }$ (500 knots for horizontal speed, $300 \mathrm{ft} /$ minute for vertical speed). Then, the variance becomes

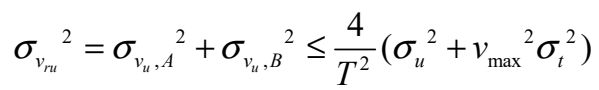

The standard deviation can be bounded by

$$
\sigma_{v_{r u}} \leq \frac{2 \sigma_{u}}{T} \sqrt{1+\frac{v_{\max }^{2} \sigma_{t}^{2}}{\sigma_{u}^{2}}}
$$

Finally, each variance in Eq. (22) can be expressed by the variance of the position measurement error. Eq.(32) can be further simplified. As an example, taking the upper bound as $v_{\max }=480 \mathrm{kt}(=0.133 \mathrm{NM} / \mathrm{s})$ in place of using $\hat{v}_{u}$ yields the condition $v_{\max } \sigma_{t} / \sigma_{u}=1.33 \times 10^{-3}<<1$ for $\sigma_{t}$ $=10^{-3} \mathrm{~s}, \sigma_{u}=\sigma_{x}=\sigma_{y}=0.1 \mathrm{NM}$ ( e.g. a typical accuracy requirement for both along and across trajectory positions is $170 \mathrm{~m}$ ( $\mathrm{rms}$ errors) [16]). This means that the terms of temporal error $\sigma_{t}^{2}$ are negligibly small except in the vicinity of $\sigma_{u}=0$.

\section{Example of Calculated Result}

To grasp the characteristics of the estimation error of the difficulty value with regards to the encounter conditions of aircraft pairs, the propagation errors of input uncertainties are investigated for en-route cruise flight phase. The scale parameters used for calculating the difficulty values are $\lambda_{H}=6 \mathrm{NM}, \lambda_{z}=1,200 \mathrm{ft}$ and $\lambda_{t}=360 \mathrm{~s}$.

Fig. 2 shows the geometry of an aircraft pair encounter used for calculation. At a given time under consideration, aircraft $\mathrm{A}$ and $\mathrm{B}$ are separated by a distance $R_{r}$ and fly linearly at the same altitude and constant speeds $V_{a}(=480 \mathrm{kt})$ and $V_{b}=V_{a}+\Delta V$, respectively. The azimuths of $\mathrm{A}$ and $\mathrm{B}, \theta_{b}$ and $\theta_{a}$, were varied within the range $[0,360]$ degrees at 5-degree intervals, and the difficulty value $G\left(t_{0}\right)$ and its propagation error $\sigma_{G}$ were calculated for each case.

The difficulty value of Eq. (13) is determined by the relative distance between an aircraft pair on diverging 
trajectory at the time of observation $t_{0}$. For the converging case, the value $G\left(t_{0}\right)$ depends on not $t_{0}$ but $t_{\min }$.

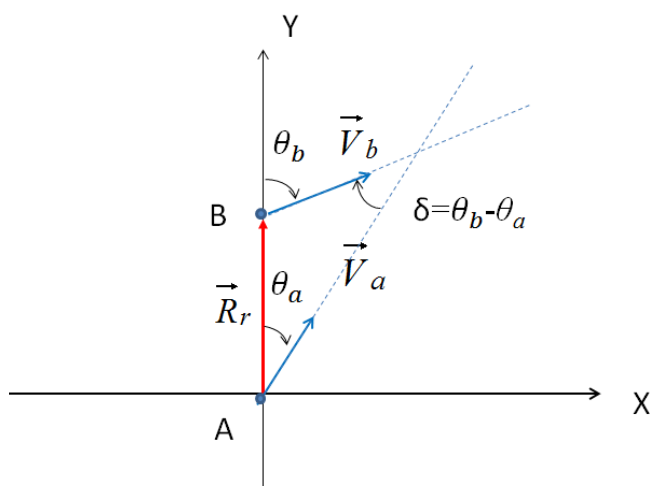

Fig. 2 Geometry of an encounter between aircraft A and B

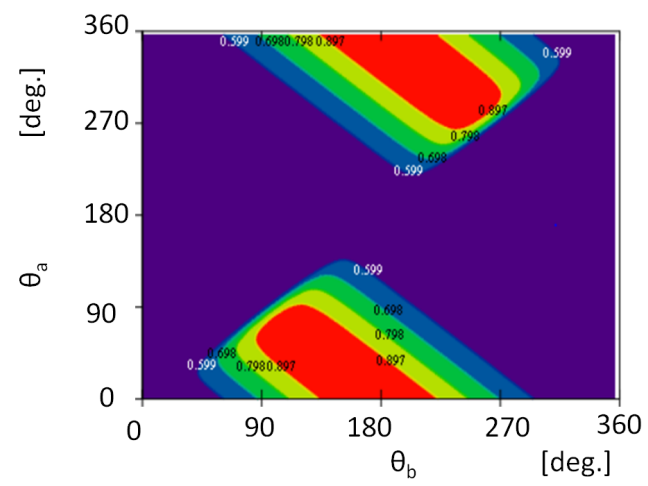

Fig. 3 Distribution of difficulty values $\left(R_{r}=5 \mathrm{NM}, \Delta \mathrm{V}=0, \max .=1.0, \min .=0.5\right)$

Fig. 3 shows 5-levels contours of the difficulty value for $R_{r}=5 \mathrm{NM}, \Delta V=0 \mathrm{kt}$. Difficulty values are distributed in the interval $[0.5,1.0]$. The minimum value in the azurecolored areas is 0.5 and maximum value in the red areas is 1.0. The distribution is rotationally symmetric with respect to the point $(0,0)$. Difficulty values of 1.0 and 0.5 correspond respectively to a collision between $\mathrm{A}$ and $\mathrm{B}$ and a "conflict" (where prescribed vertical and/or horizontal separation minima are infringed). In this case, points that make up the high difficulty ridge are in the direction of $\theta_{a}=\pi-\theta_{b}$. On the line of $\theta_{a}=\theta_{b}$, the headings of the two aircraft are the same, and the difficulty value is determined only by the current relative distance.

Fig. 4 shows contours of difficulty value $G\left(t_{0}\right)$ divided into 10 intervals between minimum and maximum values. The red and azure-colored regions contain the maximum and minimum values, respectively. The value ranges of the azure regions in Fig. 4 depend on $R_{r}$. The ranges of level variation for $R_{r}$ values of 5,15 and $40 \mathrm{NM}$ are $[0.5,0.99],[0,0.98]$ and $[0,0.84]$, respectively.

The contours on the left hand side (LHS) of Fig. 4 show distributions of $G\left(t_{0}\right)$ for $R_{r}=5 \mathrm{NM}$ while varying the speed difference $\Delta V$ between the aircraft. The horizontal axis shows $\theta_{b}$ and the vertical axis shows $\theta_{a}$. Fig. 4 indicates that the red area (the highest level) shrinks as $R_{r}$ increases. $\tau$ becomes larger as $R_{r}$ increases, resulting in lower difficulty values. This is a characteristic of Eq. (13). Variation of relative speed changes the shape of the distributions since it shifts the time of the proximity event for the aircraft pair.

Fig. 5 shows the distributions of the standard deviation (s.d.) of propagation errors $\sigma_{G}$ calculated by the method presented in section 3.3.2 (i.e., Eq. (22)). Errors of input data are assumed to have zero bias and a constant standard deviation. $\sigma_{x}=\sigma_{y}=\sigma=0.1 \mathrm{NM}, \sigma_{z}=100 \mathrm{ft}$, and $\sigma_{t}=10^{-3} \mathrm{sec}$ are assumed.

The LHS of Fig. 5 shows cases where only $\Delta V$ is varied for $R_{r}=5 \mathrm{NM}$, and the RHS cases for $\Delta V=0 \mathrm{kt}$ and $R_{r}=15 \mathrm{NM}$ or $R_{r}=40 \mathrm{NM}$. The conditions of $R_{r}$ and $\Delta V$ correspond to those in Fig. 4. These indicate that $\Delta V$ changes the shape of the difficulty contours and the change in $R_{r}$ changes the location of the high level region.
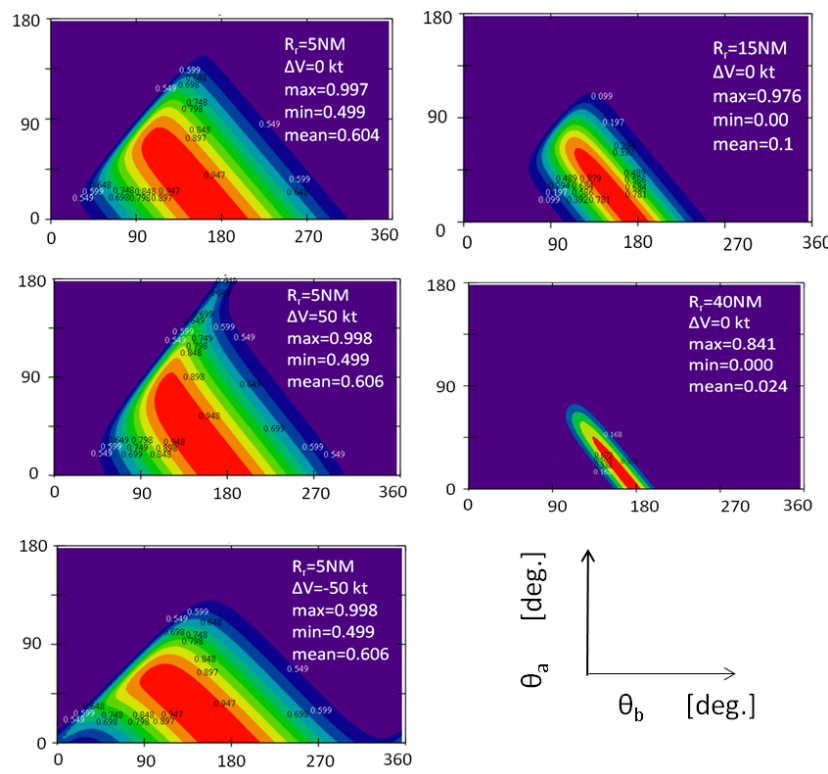

Fig. 4 Distribution of difficulty value $G\left(t_{0}\right)$ (abscissa: $\theta_{b}$, ordinate: $\theta_{a}$ ) 

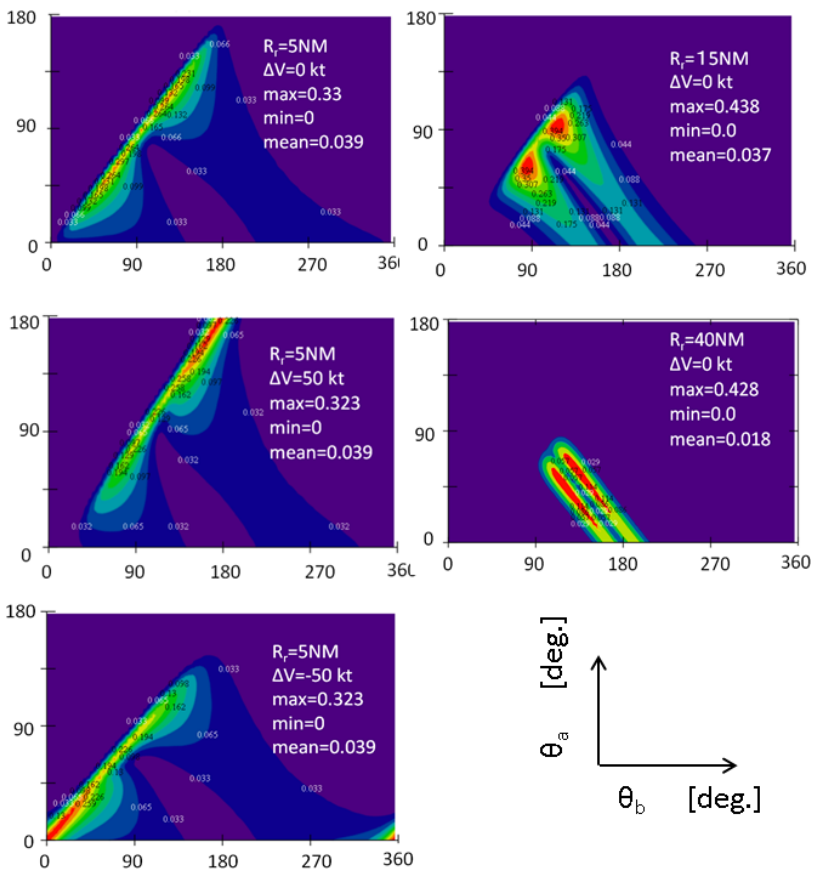

Fig. 5 Distribution of propagation errors $\sigma_{G}$

$$
\left(\sigma=\sigma_{x}=0.1 \mathrm{NM}, \sigma_{z}=100 \mathrm{ft}\right)
$$

For the case of $R_{r}=5 \mathrm{NM}$, a region with relatively high values of $\sigma_{G}$ is observed along and adjacent to the line $\theta_{b}=\theta_{a}$. In these cases, the time to the proximity event $(\tau)$ is fairly large. Since some of the partial derivatives in Table 1 contain $\tau$ in addition to the relative position and velocity components, the influences of these terms may have appeared. $\sigma_{G}$ is approximately zero in the region where $G\left(t_{0}\right)$ is very high (close to 1 ).

In Eq. (22), as $\tau$ increases, the term of the differential coefficient increases but the term of the exponential function (i.e. $G\left(t_{0}\right)$ ) decreases. As a result of these effects canceling out, an extremum is created. This seems to be the reason why a region with a relatively high $\sigma_{G}$ is created outside the high difficulty region so as to surround it.

To examine the relationship between the s.d. of the input and resulting propagation errors, the graphs in the lower part of Fig. 6 plot the distributions of $\sigma_{G}$ for two cases: $\sigma=0.1 \mathrm{NM}$ and $0.033 \mathrm{NM}$. The results show that $\sigma_{G}$ is roughly proportional to the magnitude of $\sigma$. This is also suggested by Eq.(22) if the geometic configuration of aircraft pairs is the same.

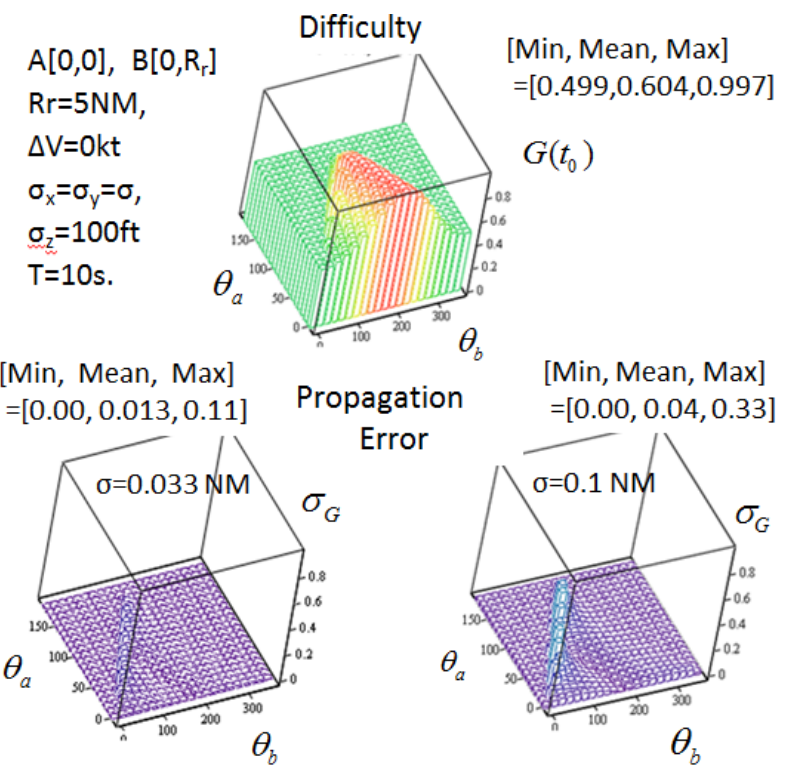

Fig. 6 Comparison of propagation error distributions

$$
\left(\sigma_{x}=\sigma_{y}=\sigma\right)
$$

\section{5 . Global Sensitivity Analysis}

\subsection{Situations for Sensitivity Analysis}

Observed position and velocity are represented by several parameters. This section examines how each parameter affects the calculated difficulty value by a sensitivity analysis using the Sobol indices [17], which consider conditional variance as an index.

Of all possible encounter geometries between a pair of aircraft, only converging cases are of relevance to our difficulty index. This paper deals with the converging situations for sensitivity analysis using the scenario model developed by Huo et al [19]. An aircraft pair A and B are assumed to be in level flight at the same altitude at constant speeds. The model uses the intersection point of the aircraft trajectories as the origin $\mathrm{O}$ of a coordinate system as shown in Fig. 7, which shows the geometry of an encounter at an instant in time and can be regarded as a sample taken from a population of all possible encounter geometries. Aircraft A and B are located inside an airspace shown as a circle of radius $R$. The $\mathrm{Y}$ axis is the flight direction of aircraft $\mathrm{A}$ which is fixed at $(0, R)$. It is assumed that aircraft $B$ is located in the shaded semicircle to the left of aircraft A's trajectory to avoid the duplication of scenarios and to reduce computation cost. The pair is horizontally separated by the relative distance $\left|\vec{R}_{r}\right| \quad(R=40 \mathrm{NM}, r$ is a variable) at a given time.

Herein, four variables are chosen, namely, $r, \delta, V_{a}$ and $V_{b}$. We examine the variances of difficulty values 
for all possible encounters as a function of these variables.

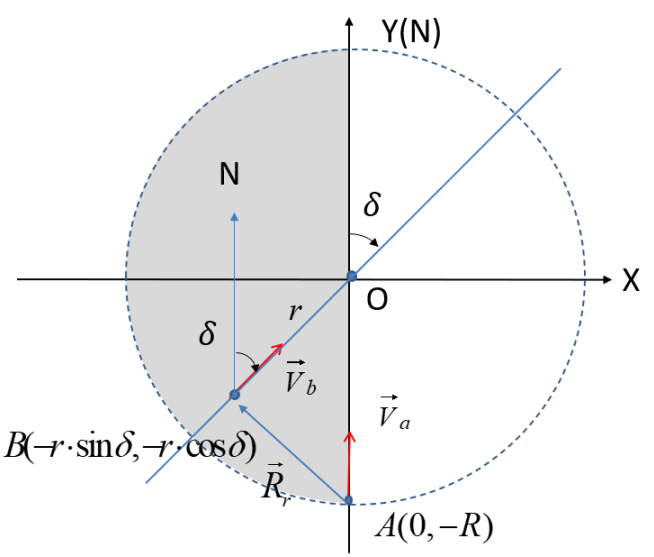

Fig. 7 Geometric configuration of aircraft pair A, B.

\subsection{Calculation of Sobol Indices}

Sobol indices $[17,18]$ have previously been applied to a conflict problem [19] which is similar to the present problem. We apply the indices to the model function $Y$ (= $\left.G\left(t_{0}\right)\right)$ assuming that $Y$ is given by

$$
Y=f\left(x_{1}, x_{2}, \ldots, x_{k}\right)
$$

where input variables $X_{1}, X, \cdots, X_{k}$ are independently and uniformly distributed within a unit hypercube (i.e., $X_{i} \in[0,1]$ for $\left.i=1,2, \cdots, k\right)$. The input space of the difficulty value can be transformed into this unit hypercube. Then, the Sobol indices are given by [17] as

$$
\begin{aligned}
& S_{i}=\frac{V_{x_{i}}\left(E_{x_{\sim i}}\left(Y \mid X_{i}\right)\right)}{V(Y)} \\
& S_{T_{i}}=\frac{E_{x_{\sim i}}\left(V_{x_{i}}\left(Y \mid X_{\sim i}\right)\right)}{V(Y)}
\end{aligned}
$$

where the subscript $\sim i$ indicates a set of all variables except $i . S_{i}$ is the first-order sensitivity index and shows the contribution to the output variance of the main effect of $X_{i} . S_{T_{i}}$ is the total-effect index which measures the contribution to the output variance of $X_{i}$, including all variance caused by its interactions, of any order, with any other input variables. $V(Y)$ is the variance of $Y$.

The function does not seem to be analytically tractable for evaluating the indices (integrals). In this case, they are estimated by the Monte Carlo method. The amount of calculation necessary increases with the number of variables $k$. For example, if the sample size is $N$ and the number of variables is $k, N^{k-1}$ and $N^{k}$ of additions may be required to compute $E_{x_{\sim i}}\left(Y \mid X_{i}\right)$ and $S_{i}$, respectively. To reduce this computational burden, an approximated calculation was carried out as described below.
5.3 Procedure of Monte Carlo simulation

The approximated computation by the Monte Carlo method generates a sequence of randomly-distributed points inside the hypercube $[17,18]$. The procedure is as follows .

(1) Generate a $N \times 2 k$ sample matrix. Each row is a sample point in a hypercube of $2 k$ dimensions. This was done with respect to the distributions of the input data $u_{i}$.

(2) Use the first $k$ columns of the matrix as $A$, and the remaining $k$ columns as matrix $B$. This gives two independent samples of $N$ points in the unit hypercube .

(3) Construct $k$ sets of $N \times k$ matrices $A_{B}^{(i)}$ ( $i=1,2, \cdot \cdot k)$. The $j$-th column of each matrix is equal to the $i$-th columns of $B$ for $j=1,2, \cdots, k$ and rest of the columns are from $A$.

(4) Matrices $A, B$ and the $k$ sets of $A_{B}^{(i)}$ specify a total of $N \times(k+2)$ points in the input space (one for each row). Compute the values of $f(A), f(B)$, $f\left(A_{B}^{(i)}\right)$ of the model for each row of the matrices $A$, $B, A_{B}^{(i)}$ (in total, $N \times(k+2)$ points) .

(5) The Monte Carlo estimators used for both indices [18] are

$$
\begin{aligned}
& V_{x_{i}}\left(E_{x_{\sim i}}\left(Y \mid x_{i}\right)\right) \approx \frac{1}{N} \sum_{j=1}^{N} f(B)_{j}\left(f(A)_{j}-f\left(A_{B}^{(i)}\right)_{j}\right) \\
& E_{x_{\sim i}}\left(V_{x_{i}}\left(Y \mid x_{\sim i}\right)\right) \approx \frac{1}{2 N} \sum_{j=1}^{N}\left(f(A)_{j}-f\left(A_{B}^{(i)}\right)_{j}\right)^{2}
\end{aligned}
$$

where $f(A)_{j}$ is a function of which the variables are row data of the $j$-th column of matrix $A . f\left(A_{B}^{(i)}\right)_{j}$ is a function of which the variables are row data of the $j$-th column of the matrix $A$ where only the $i$-th row is replaced by that of matrix $B$.

Herein, the input $u_{i}(i=1,2, \cdots, k)$ to the function $G\left(t_{0}\right)$ corresponding to $f$ was assumed to be uniformly distributed between $\left[u_{\min , i}, u_{\max , i}\right]$ and was transformed to a value $x_{i}$ uniformly distributed between $[0,1]$ by the equation

$$
u_{i}=\left(u_{\max , i}-u_{\min , i}\right) x_{i}+u_{\min , i}
$$

\subsection{Example of computation}

The input variables chosen are the intersection angle $u_{1}=\delta$, the distance of aircraft B from the intersection $u_{2}=r$, the speed of aircraft $\mathrm{A} u_{3}=V_{a}$, and the speed of aircraft B $u_{4}=V_{b} \quad(k=4)$. All variables are assumed to be uniformly distributed.

Table 2 shows the results of five estimation (simulation) trials for the $1^{\text {st }}$ order indices $S_{i}$. Table 3 show the results for the total effect index. We have $S_{1}>S_{2} \gg$ $S_{4} \approx S_{3}$ for first order effects and $S_{T 1}>S_{T 2} \gg S_{T 4} \approx S_{T 3}$ for 
total effects. The order for the corresponding variables is $\delta>r>V_{b} \approx V_{a}$ for both indices.

The results in Tables 2 and 3 suggest that the effects of variable $x_{1}$ (corresponding to the intersection angle $\delta$ ) and $x_{2}$ (corresponding to the distance of aircraft B from the intersection $r$ ) are predominant on the difficulty value ( $Y$ ). The effects of the aircraft speeds seem to be relatively small. These tendencies can be recognized from the distributions of difficulty values shown in Figs. 3-6.

Table $21^{\text {st }}$-order indices $\quad\left(\delta=[0,180]\right.$ deg. , $\mathrm{r}=[0,40] \mathrm{NM}, \mathrm{V}_{\mathrm{a}}$, $\left.\mathrm{V}_{\mathrm{b}}=[430,530] \mathrm{kt}, \mathrm{N}=100,000\right)$

\begin{tabular}{|c|c|c|c|c|c|c|}
\hline $\begin{array}{c}\text { Trial } \\
\text { No }\end{array}$ & $\mathrm{S}_{1}$ & $\mathrm{~S}_{2}$ & $\mathrm{~S}_{3}$ & $\mathrm{~S}_{4}$ & $\mathrm{~V}(\mathrm{Y})$ & $\mathrm{E}(\mathrm{Y})$ \\
\hline 1 & 0.488 & 0.317 & 0.007 & 0.016 & 0.053 & 0.18 \\
\hline 2 & 0.502 & 0.319 & 0.008 & 0.017 & 0.053 & 0.18 \\
\hline 3 & 0.504 & 0.313 & 0.005 & 0.018 & 0.053 & 0.18 \\
\hline 4 & 0.491 & 0.318 & 0.006 & 0.016 & 0.053 & 0.18 \\
\hline 5 & 0.495 & 0.318 & 0.006 & 0.016 & 0.053 & 0.18 \\
\hline
\end{tabular}

Table 3 Total-effect index $(\delta=[0,180]$ deg. , $\left.\mathrm{r}=[0,40] \mathrm{NM}, \mathrm{V}_{\mathrm{a}}, \mathrm{V}_{\mathrm{b}}=[430,530] \mathrm{kt}, \mathrm{N}=100,000\right)$

\begin{tabular}{|c|c|c|c|c|c|c|}
\hline $\begin{array}{c}\text { Trial } \\
\text { No }\end{array}$ & $\mathrm{S}_{\mathrm{T} 1}$ & $\mathrm{~S}_{\mathrm{T} 2}$ & $\mathrm{~S}_{\mathrm{T} 3}$ & $\mathrm{~S}_{\mathrm{T} 4}$ & $\mathrm{~V}(\mathrm{Y})$ & $\mathrm{E}(\mathrm{Y})$ \\
\hline 1 & 0.630 & 0.477 & 0.033 & 0.047 & 0.053 & 0.18 \\
\hline 2 & 0.631 & 0.484 & 0.033 & 0.048 & 0.053 & 0.18 \\
\hline 3 & 0.631 & 0.479 & 0.032 & 0.048 & 0.053 & 0.18 \\
\hline 4 & 0.631 & 0.481 & 0.032 & 0.048 & 0.053 & 0.18 \\
\hline 5 & 0.631 & 0.480 & 0.032 & 0.048 & 0.053 & 0.18 \\
\hline
\end{tabular}

\section{Summary and Concluding Remarks}

For a proposed index of air traffic control difficulty based on encounters between pairs of aircraft, this paper examined the relationship between uncertainty (error) in the inputs and uncertainty in the calculated difficult metric by two sensitivity analysis evaluations for a pair of aircraft in cruising flight phase at the same altitude at constant speeds: an analysis of how error in the input data is propagated to the calculated index, and a variance-based approach to determine the sensitivity of the index to each parameter that characterizes the encounter geometry.

In the former, the formula for the propagation of error at a certain point was derived based on an assumed input error. The formula enabled estimation of the variance of the propagation errors. The distribution of propagation error was investigated for all possible situations and compared with the distribution of difficulty values, and its characteristics were clarified.

The latter is a variance-based sensitivity analysis that evaluates the effect of input parameters using conditional variances called the Sobol indices. Using a Monte Carlo method, we investigated the effect of input variables (parameters) on the difficulty value for all possible situations of aircraft pairs with intersecting trajectories. The results demonstrated that the most influential parameter on the difficulty value is the intersection angle of trajectories.
The analyses of this paper are based on several assumptions. When using the difficulty index in a practical application, further careful consideration on the assumptions taking into account the characteristics of errors in the input data may be required.

\section{References}

[1] M. Brown et al, "Full 4D Trajectory Based Operation Concept Study," Proc. of APISAT (Asia Pacific Int'l Symposium on Aerospace Technology) 2013, 04-05-02, Takamatsu, Nov. 2013.

[2] B. Hilburn, "Cognitive Complexity in Air Traffic Control- A Literature Review," EEC Note No.04/04, EUROCONTROL, March 2004

[3] M. Prandini et al, "Toward Air Traffic Complexity Assessment in New Generation Air Traffic Management Systems," IEEE Trans. on Intelligent Transportation Systems, vol.12, no.3, pp.809-818, 2011 .

[4] D. Delahaye and S. Puechmorel, "Air Traffic Complexity: Towards Intrinsic Metrics," The $3^{\text {rd }}$ ATM Seminar, Napoli, June 2000.

[5] S. R. Kinnersly and I. Wilson, "INTEGRA Metrics \& Methodologies Safety Metrics Technical Definitions," EUROCONTROL, Nov. 2000.

[6] R. Gingell, C. Strachan, A. Taylor, S. Kinnersly., and S. Fox, "INTEGRA Metrics \& Methodologies Execution Phase- Final Report," Edition 1.0, EUROCONTROL, Nov. 2004.

[7] X. Zhu, X. Cao, K. Cai, "Measuring air traffic complexity based on small samples", Chisese Journal of Aeronautics, vol.30, no.4, pp.1493-1505, June 2017.

[8] H. Wang ,Z. Song, and R. Wen, "Modeling Air Traffic Situation Complexity with a Dynamic Weighted Network Approach," Journal of Advanced Transportation, vol.2018, Article ID 5254289, 15 pages, https://doi.org/10.1155/2018/5254289, Jan. 2018.

[9] H. J. Wee, S.W. Lye, and J-P Pinhiro, "A Spatial, Temporal Complexity Metric for Tactical Air Traffic Control," Journal of navigation, vol.71, pp.1040-1054, May 2018.

[10] S. Nagaoka and M. Brown, "A Review of Safety Indices for Trajectory-Based Operation in Air Traffic Management," Trans. JSASS Aerospace Tech. Japan, vol. 12, no. APISAT-2013, pp. a43-a49, 2014

[11] S. Nagaoka, and M. Brown, "Constructing an Index of Difficulty for Air Traffic Control Using Proximity Parameters," Procedia Engineering, 99 (2015), pp.253-258, Feb. 2015.

[12] S. Nagaoka, H. Hirabayashi, and M. Brown, "Method for Scale Parameter Determination of Air Traffic Control Difficulty Index Based on Survey Results of Controller's Recognition for Aircraft Proximities," 2016 Asia-Pacific International Symposium on Aerospace Technology, Toyama, Japan, Oct. 2016.

[13] S. Nagaoka. and M. Brown, "Tuning the Temporal Parameter of ATC Difficulty Metrics Using Controller's Cognitive Thresholds and Trajectory Change Point Information,” 2017 Asia-Pacific International Symposium on Aerospace Technology, Seoul, Korea, Oct. 2017.

[14] S. Nagaoka, and M. Brown, "Predicting Trajectories with Course Change points for Calculating Proximity-based Air Traffic Control Difficulty Indices," IEICE Technical Report, SANE2017-79, Nov. 2017.

[15] S. Nagaoka, H. Hirabayashi, and M. Brown, "A Concept of Automatic Trajectory Modification for Mitigating Complex Air Traffic Situations with a High Difficulty Index Value," IEICE Technical Report, SANE2018-80, Nov. 2018.

[16] EUROCONTROL Standard for Radar Surveillance in En-Route Airspace and Major Terminal Areas,1 march 1997. https://www.eurocontrol.int/publication/eurocontrol-standard- 
radar-surveillance-en-route-airspace-and-major-terminal-areas

[17] I. M. Sobol, "Global sensitivity indices for nonlinear mathematical models and their Monte Carlo estimates," Mathematics in Simulation, vol.55 no.1, pp.271-280, 2001.

[18] A. Saltelli et al., "Variance based sensitivity analysis of model output. design and estimator for the total sensitivity index," Computer physics communications, vol.181, pp.269-270, 2010.

[19] Y. Huo, D. Delahaye, and Y. Wang, "Sensitivity Analysis of Closest Point of Approach," International Conference for Research in Air Transportation (ICRAT) 2018, Barcelona, Spain, July 2018.

[20] V. D. Hopkin, Human Factors in Air Traffic Control, AGFARDograph No.275, AGARD, 1982.

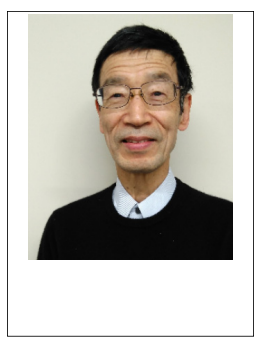

Sakae Nagaoka is a visiting researcher at the Electronic Navigation Research Institute (ENRI). He received the B.Eng. and M.Eng. degrees in electronic engineering from the University of Electro-communications in 1972 and 1974, respectively. He received the Dr. of Eng. degree in electrical engineering from the University of Tokyo in 1986. From 1974-2006 he worked for ENRI as a researcher, manager and division director. He was a visiting professor at the University of Tokyo, and Tokyo University of Marine Technology and Science. He is a Fellow of the Royal Institute of navigation, and Japan Society for Aeronautical and Space Sciences.

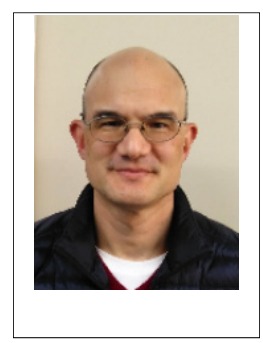

Mark Brown received a B.Eng. degree in Avionics in 1990 and a Ph.D. in Computer Science in 1996 from Queen Mary, University of London. He was a post doctorate research fellow at the National Aerospace Laboratory (now JAXA) and ENRI between 1996 and 2000 looking at human factors and cockpit displays, before joining $\mathrm{OKI}$ as a systems engineer specializing in Aeronautical Telecommunication Network (ATN) systems. He re-joined ENRI in 2010 as a researcher in ATM. He has contributed to ICAO, RTCA and EUROCAE standardization activities including ATM, ATN and remote tower.

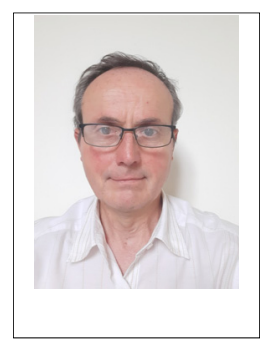

Daniel Delahaye is a professor and the head of the Optimization and Machine Learning team at ENAC LAB (French Civil Aviation University). He is also in charge of the new research chair: "AI for ATM and Large Scale Urban Mobility" in the new AI institute in Toulouse. ANITI. He obtained his engineer degree from the ENAC school and did a master of science in signal processing from the national polytechnic institute of Toulouse in 1991. He obtained his Ph.D. in automatic control from the aeronautics and space national school in 1995 and did a post-doc at the Department of Aeronautics and Astronautics at MIT in 1996. He gets his tenure in applied mathematics in 2012. He conducts researches on optimization and on machine learning with applications to air traffic management (airspace design, trajectory planning, and clustering, sequencing and merging, airport, oceanic traffic optimization, etc...). 